\title{
Phonological Subcategorization, Infixation, and Relation-Specific Alignment
}

\author{
Brett Hyde and Jonathan Paramore \\ Washington University in Saint Louis
}

In the Phonological Subcategorization approach to infixation (Kiparsky 1986, McCarthy and Prince 1986, Inkelas 1990, Anderson 1992, Yu 2007), the position of an infix is determined by locating it at the appropriate edge of a particular phonological category, or "pivot". The basic pattern of Tagalog -um- infixation provides a simple illustration. In Tagalog, the affix -um- is an agentive focus marker. It anchors itself to the right edge of the stem-initial consonant or, optionally, to the right edge of the stem initial onset. In the former case, the pivot is consonant; in the latter, the pivot is onset.

(1) Tagalog forms (Orgun and Sprouse 1999)

Initial Single Consonant
a. sulat s-um-ulat
'to write'
b. Pabot P-um-abot
'to reach for'
Initial Cluster
c. gradwet g-um-radwet $\sim$ gr-um-adwet
d. preno p-um-reno $\sim$ pr-um-eno
'to graduate'
'to brake'

Relation-Specific Alignment (RSA; Hyde 2012, 2015, 2016) provides a natural and effective approach to Phonological Subcategorization. RSA constraints are based on one of the three general schemas given in (2). In the RSA schemas, ACat1 and ACat2 are the aligned categories. SCat is the separator category, the category whose intervention between the relevant edges of ACat 1 and ACat 2 constitutes misalignment. The configuration to the right of the slash in the individual schemas is the configuration of misalignment prohibited by that schema. The set of categories to the left of the slash defines the locus of violation. A violation is assessed each time the categories that define the locus occur in the prohibited configuration. When SCat is included in the definition of the locus of violation, violation assessment is distance-sensitive: as the degree of misalignment increases, the number of violations assessed also increases. When SCat is omitted from the definition of the locus of violation, violation assessment is distance-insensitive: a single violation is assessed for each pair of misaligned edges, regardless of the degree of misalignment.

Relation-Specific Alignment constraint schemas
a. Left-Edge:
* $\langle$ ACat 1, ACat $2,($ SCat $)\rangle /[\text {... SCat ... ACat } 2 \ldots]_{\text {ACat } 1}$ 'Assess a violation mark for every $\langle A C a t 1, A C a t 2,(S C a t)\rangle$ such that SCat precedes ACat2 within ACat1.'
b. Right-Edge: * $\langle$ ACat 1, ACat $2,($ SCat $)\rangle /[\text {... ACat } 2 . . \text { SCat ... }]_{\text {ACat } 1}$ 'Assess a violation mark for every $\langle A C a t 1, A C a t 2,(S C a t)\rangle$ such that ACat2 precedes SCat within ACat1.'
c. Opposite-Edge: ACat1 precedes SCat and SCat precedes ACat2.'

Though RSA constraints can be either distance-sensitive or distance-insensitive, they are always relation-specific: they only penalize misalignment when the misaligned categories occur in the structural configuration indicated. The schema in (2a), for example, prohibits misalignment between the left edges of ACat 2 and $A C a t 1$ by prohibiting $S C$ at from preceding $A C a t 2$ within ACat1. It only prohibits misalignment, however, when ACat1 contains ACat2. Schema (2b) prohibits misalignment of right edges, but only when 
ACat 1 contains ACat2. Finally, schema (2c) prohibits misalignment between the right edge of ACat 1 and the left edge of $A C a t 2$, but only when ACat1 precedes ACat 2 .

To demonstrate the effectiveness of RSA constraints as an approach to infixation, I examine them in the context of Yu's (2007) Phonological Subcategorization framework, replacing Yu's original Generalized Alignment (GA; McCarthy and Prince 1993) constraints with RSA constraints. RSA constraints preserve the framework's essential successes, but they also offer several advantages over GA constraints.

\title{
1 An RSA Approach
}

In Phonological Subcategorization, infixes attach to a particular pivot. In the proposed approach, any one of the categories in (3) can be a pivot simply by being designated as an argument in a non-violable subcategorization constraint. Subcategorization constraints are always formulated as RSA constraints.

Category pivots
Vowel
Consonant
Onset
Syllable

\author{
Prominence Pivots \\ Stressed vowel \\ Stressed syllable \\ Stressed foot
}

The case of Tagalog -um- infixation is a useful example because it illustrates infixation targeting two different pivots: consonant and onset. In circumstances where consonant is the pivot, the opposite-edge RSA constraint $u m$-C-PIVOT, given in (4a), is the non-violable subcategorization constraint. It restricts the infix to a position adjacent to the initial consonant by prohibiting a segment from intervening between -umand any consonant to its left. In circumstances where onset is the pivot, the non-violable subcategorization constraint is $u m$-ONS-PIVOT, given (4b). um-ONS-PIVOT restricts the infix to a position adjacent to the initial onset by prohibiting a segment from intervening between -um- and any onset to its left.

Tagalog subcategorization constraints

a. um-C-Pivot: ${ }^{*}\langle C$, um, seg $\rangle / C \ldots$ seg $\ldots u m$

'Assess a violation mark for every $\langle C, u m$, seg $\rangle$ such that a consonant precedes -um- with a segment intervening.'

b. um-ONS-PIVOT: *〈ons, um, seg $\rangle /$ ons ... seg ... um

'Assess a violation mark for every $\langle o n s, u m$, seg $\rangle$ such that an onset precedes -um- with a segment intervening.'

The difference between the two subcategorization constraints can most easily be seen when considering forms with potentially complex initial onsets, forms where the initial onset is potentially larger than just the initial consonant. As (5) illustrates using [gradwet] 'graduate', a form whose stem begins with a consonant cluster might ultimately have no initial onset, as in candidate (a), a simple initial onset, as in candidate (b), or a complex initial onset, as in candidates (c-f), depending on the position of the infix. Both um-CPIVOT and um-ONS-PIVOT establish a window of positions in which the infix might occur, but um-ONSPIVOT establishes a larger window than $u m$-C-PIVOT because the initial onset is potentially larger than just the initial consonant.

$u m$-C-PIVOT restricts the infix to a position just before or just after the initial consonant. When it occurs in either of these positions, there is no segment between the infix and a consonant to its left, either because there is no consonant to the infix's left, as in (5a), or because the left edge of the infix coincides with the left edge of the initial consonant so that no segment can intervene, as in (5b). In contrast, um-ONSPIVOT establishes a slightly larger window. It allows the infix to occur on either side of the initial consonant, just like um-C-PIVOT, but it also allows it to occur just after the second consonant, as in (5c). When the infix occurs in any of these three positions, there is no segment between the infix and an onset to its left, either because there is no onset to the infix's left, as in (5a), or because the left edge of the infix coincides with the right edge of the initial onset so that no segment can intervene, as in $(5 b, c)$. The difference between $(5 b)$ and $(5 c)$ is that the initial onset in (5b) is simple while the initial onset in (5c) is complex. 
(5)

\begin{tabular}{|c|c|c|}
\hline um + gradwet & um-C-PIVOT & $u m$-ONS-PIVOT \\
\hline a. um.grad.wet & $\Leftrightarrow$ & $\Leftrightarrow$ \\
\hline b. gum.rad.wet & $\Leftrightarrow$ & 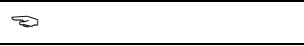 \\
\hline c. gru.mad.wet & $*$ & $\Leftrightarrow$ \\
\hline d. gra.dum.wet & $* * * * *$ & $* *$ \\
\hline e. grad.wu.met & $* * * * * * * *$ & $* * *$ \\
\hline f. grad.we.tum & $* * * * * * * * * * * * * * * * *$ & $* * * * * * *$ \\
\hline
\end{tabular}

Whether the pivot is consonant or onset, an additional, violable constraint is necessary to fix the affix's position within the window established by the subcategorization constraint. In the Tagalog case, ALIGN$u m$-RIGHT, given in (6), ensures that the infix occurs as far to the right within the window as possible. ALIGN-um-RIGHT is a right-edge RSA constraint prohibiting -um- from preceding a segment within a stem.

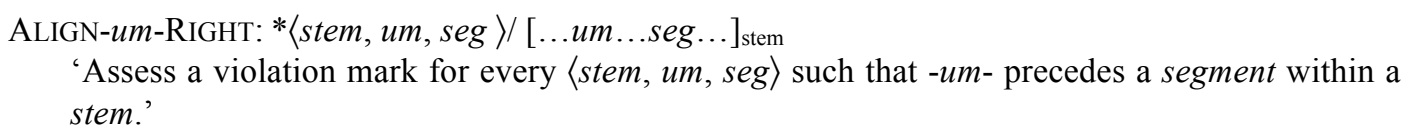

To illustrate, in (7) and all subsequent tableaux, non-violable constraints appear to the left of a vertical double line and all candidates excluded by a non-violable constraint appear below a horizontal double line. Consider, first, forms where the initial onset can only be simple — when the stem is [sulat] 'to write', for example - and the choice of either consonant or onset as pivot yields the same result. When the relevant pivot is consonant, the non-violable um-C-PIVOT confines -um- to a position either preceding the initial consonant, as in (7a), or immediately following the initial consonant, as in (7b). Candidates where -umoccurs in any other position are excluded. While the violable ALIGN-um-RigHT does not have enough influence to draw the infix to the right edge of the stem, it does have sufficient influence to determine the location of the infix within the window of positions allowed by $u m$-C-PIVOT. It prefers that the infix appear as far to the right within the window as possible, locating it to the right of the initial consonant.

\begin{tabular}{|c|c|c|}
\hline um + sulat & $u m$-C-PIVOT & ALIGN-um-RIGHT \\
\hline a. um.su.lat & & $* * * * * !$ \\
\hline b. su.mu.lat & & $* * * *$ \\
\hline \hline c. su.lu.mat & $* ! ! *$ & $* *$ \\
\hline d. su.la.tum & $* ! ! * * * * *$ & \\
\hline
\end{tabular}

Since the initial onset is coextensive with the initial consonant, an onset pivot yields the same result. The non-violable $u m$-ONS-PIVOT confines -um- to a position either preceding the initial consonant, as in (8a), or immediately following the initial consonant, as in (8b). The violable ALIGN-um-RIGHT draws the infix as far to the right within this window as possible, so that it follows the initial consonant.

\begin{tabular}{|c|c|c|}
\hline um + sulat & $u m$-ONS-PIVOT & ALIGN-um-RIGHT \\
\hline a. um.su.lat & & $* * * * * !$ \\
\hline b. su.mu.lat & & $* * * *$ \\
\hline \hline c. su.lu.mat & $* ! ! *$ & $* *$ \\
\hline d. su.la.tum & $* ! ! * * * * *$ & \\
\hline
\end{tabular}

Now consider the situation where the initial onset is potentially complex. Since the initial onset is not necessarily coextensive with the initial consonant, the result when onset is the relevant pivot differs from the result when consonant is the relevant pivot. In (9), um-C-PIVOT confines -um- to a position either preceding or immediately following the initial consonant, just as it does when the onset is necessarily simple. The violable ALIGN-um-RIGHT ensures that the infix follows the initial consonant. 


\begin{tabular}{|c|c|c|}
\hline um + gradwet & um-C-PIVOT & ALIGN-um-RIGHT \\
\hline a. um.grad.wet & & $* * * * * * *$ \\
\hline b. gum.rad.wet & & $* * * * * * !$ \\
\hline \hline c. gru.mad.wet & $* ! !$ & $* * * * *$ \\
\hline d. gra.dum.wet & $* ! ! * * * *$ & $* * *$ \\
\hline e. grad.wu.met & $* ! ! * * * * * * *$ & $* *$ \\
\hline f. grad.we.tum & $* ! ! * * * * * * * * * * * * * * *$ & \\
\hline
\end{tabular}

In contrast, when onset is the relevant pivot, um-ONS-PIVOT defines a larger window of potential positions for the infix. It is satisfied when -um-occurs initially, just after the initial consonant, or just after the second consonant. When it occurs after the initial consonant, the initial onset contains just a single segment. When it occurs after the second consonant, the initial onset contains two segments. Though it cannot draw the infix to the right edge of the stem, the violable ALIGN-um-RIGHT is able to draw the infix as far to the right within the window as possible, locating it to the right of the second consonant. In this context, then, ALIGN-um-RIGHT effectively ensures that the initial onset is as large as possible.

\begin{tabular}{|c|c||c|}
\hline um + gradwet & um-ONS-PIVOT & ALIGN-um-RIGHT \\
\hline a. um.grad.wet & & $* * * * *$ ! \\
\hline b. gum.rad.wet & & $* * * * * !$ \\
\hline c. gru.mad.wet & & $* * * *$ \\
\hline \hline d. gra.dum.wet & $* ! ! *$ & $* *$ \\
\hline e. grad.wu.met & $* ! ! * *$ & \\
\hline f. grad.we.tum & $* ! ! * * * * * *$ & \\
\hline
\end{tabular}

An RSA approach to Phonological Subcategorization, then, provides a solid foundation for the analysis of infixation. Below we will see that the RSA approach retains the advantages that earlier Phonological Subcategorization approaches have over alternative approaches. We will also see, however, that the RSA approach to Phonological Subcategorization has several advantages over a GA-based approach.

\section{Advantages of Phonological Subcategorization}

Phonological Subcategorization is one of two main approaches to infixation in the recent literature. The second is Phonological Readjustment (McCarthy and Prince 1993, Orgun and Sprouse 1999, McCarthy 2003). Where Phonological Subcategorization claims that infixes target a particular phonological category to which they must be anchored, Phonological Readjustment claims that infixes occur as infixes, rather than as prefixes or suffixes, in an effort to better satisfy a phonological well-formedness constraint.

Under a Phonological Readjustment approach to Tagalog -um- infixation, for example, -um- prefers prefixation but will move rightward within a stem in order to better satisfy a phonological well-formedness constraint. A constraint like ALIGN-um-LEFT, defined in (11a) as an RSA constraint, prefers the affix to occur at the left edge of the stem. The phonological well-formedness constraint might be a constraint like ONSET, given in (11b). Faithfulness constraints like DEP-C, given in (11c), prevent alternative means, such as consonant insertion, for satisfying the phonological well-formedness constraint.

a. ALIGN-um-LEFT: * $\langle$ stem, um, seg $\rangle /[\ldots \text {...eg...um ... }]_{\text {stem }}$

'Assess a violation mark for every $\langle$ stem, um, seg $\rangle$ such that -um- follows a segment within a stem.'

b. ONSET: All syllables begin with a consonant.

c. DEP-C: Every consonant in the output is present in the input.

As (12) illustrates using [sulat] 'to write', -um- might be prefixed in two ways: it might simply attach to the beginning of the stem without any further augmentation, as in (12a), or it might attach at the beginning of the stem with a consonant inserted to its left, as in (12b). Since the former configuration leaves the 
initial syllable without an onset, it is excluded by ONSET. Since the latter configuration relies on consonant epenthesis to satisfy ONSET, it is excluded by DEP-C. There are also a number of positions in which the affix might occur stem internally. Those shown in $(12 \mathrm{c}, \mathrm{d})$ locate it after an underlying consonant, so that both satisfy ONSET with violating DEP-C. Making -um- a suffix, as in (12e), also positions it after an underlying consonant. Since (12c-e) all satisfy ONSET and DEP-C, the decision falls to ALIGN-um-LEFT. AligN$u m$-LEFT prefers $(12 \mathrm{c})$, where -um- follows the initial underlying consonant.

\begin{tabular}{|c|c|c|c|}
\hline um + sulat & ONSET & DEP-C & ALIGN-um-LEFT \\
\hline a. um.su.lat & $* !$ & & \\
\hline b. ?um.su.lat & & $* !$ & $*$ \\
\hline c. su.mu.lat & & & $* *$ \\
\hline d. su.lu.mat & & & $* *$ !*** \\
\hline e. su.la.tum & & & \\
\hline
\end{tabular}

While Phonological Readjustment produces the right result in Tagalog and many other cases of infixation, there are a number of reasons to prefer a Phonological Subcategorization approach. First, under Phonological Readjustment, it is possible for an infix to migrate to any position, not just positions near edges, where phonological well-formedness constraints might be satisfied. Hyper-infixed candidates, such as candidate (12d), are neither excluded by non-violable constraints nor harmonically bounded. There appear to be strict limits on depth of infixation (Yu 2007), however, and the non-violable subcategorization constraints of the Phonological Subcategorization approach effectively enforce these limits.

Second, Phonological Readjustment does not capture cases of infixation where the infix appears to attach to prominent positions ( $\mathrm{Yu}$ 2007). For example, the construct state markers (CSMs) in Ulwa (Green 1999) appear just to the right of the leftmost stressed syllable. With no advantage in phonological wellformedness gained from infixation, Phonological Readjustment does not account for this type. Phonological Subcategorization, however, easily handles such cases.

Ulwa construct state (Green 1999)
a. sú:lu
sú:-ma-lu
'dog- $\mathrm{CNS}_{2}$ '
b. áytak
áy-mana-tak
'paper-CNS $\mathrm{CN}_{22}$ '
c. alá:kum。
alá:-ka-kum。
d. waráwowa
waráw-kana-wa
'Muscovy duck- $\mathrm{CNS}_{3}$ '
e. ká:sirá:mah
ká:-ki-sirá:mah
'parrot sp.- $\mathrm{CNS}_{33}$ '
'lizard sp.-CNS,

To illustrate, the Phonological Subcategorization analysis could employ the non-violable subcategorization constraint in (14a) to confine the CSM to a position preceding or immediately following the leftmost stress. The additional RSA constraint in (14b) would ensure that the CSM occurred just following the stress. (In (14a), ' $X_{F}$ ' refers to the foot-level grid entry, or stress.)

a. CSM- $X_{F}$-PIVOT: $*\left\langle x_{F}\right.$, CSM, seg $\rangle / x_{F} \ldots$ seg $\ldots$ CSM

'Assess a violation mark for every $\left\langle X_{F}, \mathrm{CSM}, \operatorname{seg}\right\rangle$ such that a $X_{F}$ precedes the CSM with a segment intervening.'

b. ALIGN-CSM-Right: * $\langle$ stem, CSM, seg $\rangle /[\ldots \text {. CSM ...seg... }]_{\text {stem }}$

'Assess a violation mark for every $\langle$ stem, CSM, seg $\rangle$ such that CSM precedes a segment within a stem.'

The interaction between CSM- $X_{F}$-PIVOT and ALIGN-CSM-RIGHT is illustrated in (15). 


\begin{tabular}{|c|c||c|}
\hline CSM + ká:sirá:mah & CSM- $X_{F}-$ PIVOT & ALIGN-CSM-RIGHT \\
\hline a. ki-ká:sirá:mah & & $* * * * * * *$ !* \\
\hline b. ká:-ki-sirá:mah & & $* * * * * * *$ \\
\hline \hline c. ká:si-ki-rá:mah & $* ! ! *$ & $* * * * *$ \\
\hline d. ká:sirá:-ki-mah & $* ! ! * * *$ & \\
\hline e. ká:sirá:mah-ki & $* ! ! * * * * * * * * *$ & \\
\hline
\end{tabular}

Finally, it is difficult to see how Phonological Readjustment might be extended to cases of tmesis. There seems to be no principle of phonological well-formedness that requires the stem-internal position of bloody in abso-bloody-lutely (Yu 2007), for example. Phonological Subcategorization, however, easily handles such cases. It simply uses the combination of a subcategorization constraint and an additional alignment constraint to position the relevant structure at the desired edge of the appropriate pivot, just as it does for infixes. In the case of abso-bloody-lutely, the subcategorization constraint in (16a) and the additional RSA constraint in (16b) would locate bloody just to the left of the primary stress, as in (17). (In (16a), ' $X_{\omega}$ ' refers to the prosodic word-level grid entry, or primary stress.)

a. $\quad$ bloody-X $\mathrm{X}_{\omega}$-PIVOT: $*\left\langle\right.$ bloody, $\left.X_{\omega}, \operatorname{seg}\right\rangle /$ bloody $\ldots$ seg $\ldots X_{\omega}$

'Assess a violation mark for every $*\left\langle\right.$ bloody, $\left.X_{\omega}, \operatorname{seg}\right\rangle$ such that bloody precedes $X_{\omega}$ with a segment intervening.'

b. ALIGN-bloody-LEFT: *〈stem, bloody, seg $\rangle /[\ldots \text { seg...bloody... }]_{\text {stem }}$

'Assess a violation mark for every $\langle$ stem, bloody, seg〉 such that bloody follows a segment within a stem.'

\begin{tabular}{|c|c||c|}
\hline bloody + absolútely & bloody-X $\mathrm{X}_{\omega}$-PIVOT & ALIGN-bloody-LEFT \\
\hline a. absolútely-bloody & & $* * * *$ !**** \\
\hline b. absolúte-bloody-ly & & $* * * * *$ !** \\
\hline c. abso-bloody-lútely & & $* * * *$ \\
\hline \hline d. ab-bloody-solútely & $* ! ! *$ & $* *$ \\
\hline e. bloody-absolútely & $* ! ! * * *$ & \\
\hline
\end{tabular}

\section{Advantages of RSA Constraints}

The Phonological Subcategorization account outlined in Section 1 is in many respects the same account as that proposed by Yu (2007), and it offers the same types of advantages over Phonological Readjustment. One key difference, however, is that RSA constraints replace Yu's GA constraints. There are several advantages in replacing GA constraints with RSA constraints.

3.1 Specification of pivot categories The first advantage is specific to the analysis of infixation: the RSA approach avoids the need for complex specifications of pivot categories but maintains Phonological Subcategorization's ability to avoid hyper-infixation. For the pivots listed as category pivots in (3), it is typically necessary to provide two directional specifications to properly position an infix with respect to the pivot. First, when there is the possibility of a stem containing more than one instance of the pivot category, it is necessary to specify which instance, leftmost or rightmost, should targeted. Second, it is necessary to specify the side of the pivot, left or right, to which the infix should be anchored. The RSA formulation allows these separate specifications to be made with two separate alignment constraints, intuitively the most natural way for the specifications to be handled. In contrast, the GA formulation requires that pivot's position, leftmost or rightmost, be specified as part of the definition of the pivot itself.

In the RSA approach, the non-violable subcategorization constraint specifies the pivot's category, and the constraintss directional orientation determines which instance of the pivot category should be targeted. An additional, violable RSA constraint determines to which side of the pivot the infix occurs. In the analysis of Tagalog, for example, the non-violable subcategorization constraint um-C-PIVOT specifies the pivot category for -um- as consonant, and its directional specifications ensure that the initial consonant is targeted. Because um-C-PIVOT prohibits -um- from following a consonant with a segment intervening, it re- 
stricts -um- to a position either preceding or immediately following the initial consonant. (An RSA constraint that prohibits an infix from preceding a consonant with a segment intervening restricts the infix to a position following or immediately preceding the final consonant.) With the pivot category and position decided, the violable RSA constraint ALIGN-um-RighT determines to which side of the pivot the infix is anchored. It ensures that the infix occurs at the pivot's right edge.

In contrast, the use of GA constraints as subcategorization constraints seems to require that both directional specifications be made by a single non-violable constraint in order to avoid the possibility of hyperinfixation. To illustrate, the general formulation for GA constraints is given in (18).

\section{Generalized Alignment}

ALIGn (ACat1, Edge1, ACat2, Edge2, SCat)

The Edge1 of every ACat1 coincides with the Edge 2 of some ACat2. Assess a violation mark for every SCat that intervenes between edges that fail to coincide.

The particular GA subcategorization constraints that might be used for Tagalog -um- infixation are given in (19). The constraint in (19a) specifies initial consonant as the pivot category, incorporating the determination of the particular instance of the pivot that should be targeted into the specification of the pivot itself. The edge specifications of the constraint ensure that the infix will occur at the pivot's right edge. Similarly, the constraint in (19b) specifies initial onset as the pivot category, incorporating the determination of the targeted instance into the specification of the pivot itself.

GA subcategorization constraints

a. Align (-um-, L, $\left.\mathrm{C}_{1}, \mathrm{R}\right)$ : The left edge of every -um- coincides with the right edge of some stem-initial consonant. Assess a violation mark for each segment intervening between misaligned edges.

b. Align (-um-, L, Ons $1, \mathrm{R})$ : The left edge of every -um- coincides with the right edge of some stem-initial consonant. Assess a violation mark for each segment intervening between misaligned edges.

As (20) demonstrates, Align (-um-, L, $\mathrm{C}_{1}, \mathrm{R}$ ) picks out the first consonant, just like um-C-PIVot, but it does not require a separate constraint to ensure that -um- is located at the first consonant's right edge.

\begin{tabular}{|c|c|}
\hline um + sulat & ALIGN (-um-, $\left.\mathrm{L}, \mathrm{C}_{1}, \mathrm{R}\right)$ \\
\hline a. su.mu.lat & $* ! ! *$ \\
\hline \hline b. su.lu.mat & $* ! *^{*}$ \\
\hline c. um.su.lat & $* ! ! * * *$ \\
\hline d. su.la.tum & \\
\hline
\end{tabular}

As a result of the need to make complex specifications for pivot categories, the number of pivot categories is much larger using the GA formulation than it is using the RSA formulation. Since a directional specification that intuitiviely should be handled by something like an alignment constraint has been incorporated into the definition of a pivot category, the number of pivot categories expands accordingly.

$\begin{array}{cc} & \text { Edge pivots } \\ \text { First consonant } & \text { Final consonant } \\ \text { First vowel } & \text { Final vowel } \\ \text { First onset } & \text { Final onset } \\ \text { First syllable } & \text { Final syllable }\end{array}$

\section{Prominence pivots \\ Stressed foot \\ Stressed syllable \\ Stressed vowel}

It is, of course, possible to eliminate the specification initial from a subcategorization constraint like Align (-um-, L, $\left.\mathrm{C}_{1}, \mathrm{R}\right)$ and do the same work with a separate GA constraint, but it is not obvious how to do so in a way that avoids the possibility of hyper-infixation. For example, Align (-um-, L, $\left.\mathrm{C}_{1}, \mathrm{R}\right)$ might be 
reformulated as ALIGN (-um-, L, C, R), as in (22a), eliminating the initial specification, and an additional violable constraint aligning the left edge of the infix with the left edge of the stem, as in (22b), might be used to pick out the initial consonant.

(22) a. Align (-um-, L, C, R): The left edge of every -um- coincides with the right edge of some consonant. Assess a violation mark for each segment intervening between misaligned edges.

b. Align (-um-, L, Stem, L): The left edge of every -um- coincides with the left edge of some stem. Assess a violation mark for each segment intervening between misaligned edges.

As (23) demonstrates, the combination of the two constraints does pick out the desired candidate. The nonviolable Align (-um-, L, C, R) ensures that the infix occurs at the right edge of some consonant, as in candidates (a-c). The violable Align (-um-, L, Stem, L) is then able to ensure that it occurs at the right edge of the initial consonant, as in candidate (a).

\begin{tabular}{|c|c|c|}
\hline um + sulat & ALIGN (-um-, L, C, R) & Align (-um-, L, Stem, L) \\
\hline a. su.mu.lat & & $*$ \\
\hline b. su.lu.mat & & $* * ! *$ \\
\hline c. su.la.tum & & $* * ! * * *$ \\
\hline \hline d. um.su.lat & $* ! ! * *$ & \\
\hline
\end{tabular}

The problem with the result in (23) is that the hyper-infixation candidates $(23 \mathrm{~b}, \mathrm{c})$ are not excluded by a non-violable constraint, making it possible for them to emerge if the requirements of another higher ranking constraint were to demand it. As discussed in Section 2, its ability to eliminate hyper-infixation is one of the primary advantages claimed for Phonological Subcategorization.

3.2 Generality of the Analysis Under an RSA approach to Phonological Subcategorization, infixation receives an analysis similar to the RSA analysis of trisyllabic accent windows (Hyde 2012; Hyde, Hofmeister, and Husic 2012; Hyde and Husic 2012). In many languages, an accent is confined to the leftmost three syllables of a word or to the rightmost three syllables. Macedonian (Comrie 1976) is an example of a language with a trisyllabic window at the right edge. In Macedonian's regular accent pattern, the accent falls on the antepenult. In irregular forms, however, as illustrated in (24), accent falls on a lexically specified syllable, so long as the lexically specified syllable is one of the final three. If suffixation pushes the lexically specified syllable outside of the three-syllable window, the accent returns to the antepenult by default.

Macedonian irregular pattern

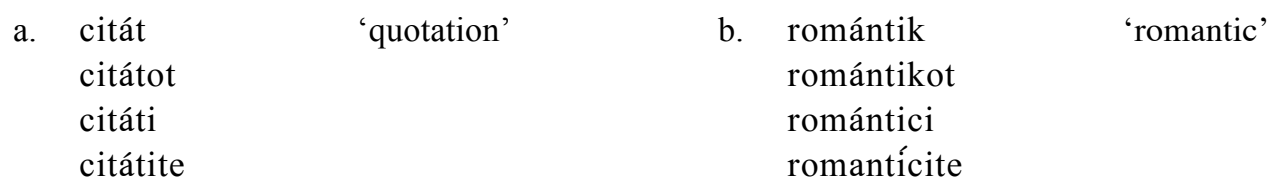

In the RSA analysis of trisyllabic accent windows, a disyllabic foot acts as a pivot for the accent in much the same way that a phonological category acts as a pivot for an infix. As (25) shows, confining an accent to a final foot or to the syllable adjacent to a final foot restricts the accent to the final three syllables.

Final foot as accent pivot

Within the window

$$
\begin{aligned}
& \sigma \sigma \sigma \sigma(\sigma \sigma) \\
& \sigma \sigma \sigma \sigma(\sigma \sigma \sigma) \\
& \sigma \sigma \sigma \sigma(\sigma \sigma)
\end{aligned}
$$
Outside the window
$\sigma \sigma \sigma ́ \sigma(\sigma \sigma)$
$\sigma \sigma \sigma \sigma \sigma(\sigma \sigma)$
$\sigma \sigma \sigma \sigma \sigma(\sigma \sigma)$

The RSA constraint responsible for establishing accent windows at the right edge of a word is FINALWINDOW, given in (26). 
FINAL-WINDOW: $*\left\langle X_{\omega}, F, \sigma\right\rangle / X_{\omega} \ldots \sigma \ldots F$

'Assess a violation mark for every $\left\langle X_{\omega}, F, \sigma\right\rangle$ such that $X_{\omega}$ precedes $F$ with $\sigma$ intervening.'

The tableau in (27) illustrates how FINAL-WINDOW establishes a trisyllabic accent window at the right edge of the word. Because it prohibits an accent from preceding a foot with a syllable intervening, FINALWINDOW is satisfied when the accent occurs within the final foot, as in $(27 \mathrm{e}, \mathrm{f})$, or at the left edge of the final foot, as in $(27 \mathrm{~d})$. If the accent occurs any further to the left, as in $(27 \mathrm{a}-\mathrm{c})$, the constraint is violated. FINAL-WINDOW, then, effectively restricts an accent to a word's final three syllables.

\begin{tabular}{|c|c|}
\hline & FINAL-WINDOW \\
\hline a. $(\sigma \sigma)(\sigma \sigma)(\sigma \sigma)$ & $* ! * * *$ \\
\hline b. $(\sigma \sigma)(\sigma \sigma)(\sigma \sigma)$ & $* ! *$ \\
\hline c. $(\sigma \sigma)(\sigma \sigma)(\sigma \sigma)$ & $* !$ \\
\hline d. $(\sigma \sigma)(\sigma \sigma)(\sigma \sigma)$ & \\
\hline e. $(\sigma \sigma)(\sigma \sigma)(\sigma \sigma)$ & \\
\hline f. $(\sigma \sigma)(\sigma \sigma)(\sigma \sigma)$ & \\
\hline
\end{tabular}

To produce the antepenultimate stress of the regular Macedonian pattern, it would simply be necessary to rank FINAL-WINDOW above the RSA constraint ACCENT-LEFT, given in (28), which aligns the accent with the left edge of the word.

$$
\begin{aligned}
& \text { ACCENT-LEFT: } *\left\langle\omega, X_{\omega}, \sigma\right\rangle /\left[\ldots \sigma \ldots X_{\omega} \ldots\right]_{\omega} \\
& \text { 'Assess a violation mark for every }\left\langle\omega, X_{\omega}, \sigma\right\rangle \text { such that } \sigma \text { precedes } X_{\omega} \text { within } \omega . '
\end{aligned}
$$

As (29) illustrates, FINAL-WINDOW confines the accent to one of the final three syllables: to one of the syllables contained within the final foot or the syllable adjacent to the final foot. The lower-ranked ACCENTLEFT draws the accent as far to the left within the window as possible, locating it on the antepenult. To obtain the Macedonian irregular pattern, it would simply be necessary to rank FINAL-WINDOW above the Faithfulness constraints responsible for maintaining the position of a lexically specified stress. See Hyde $(2012,2016)$ for more detailed discussion.

\begin{tabular}{|r|c|c|}
\hline & FINAL-WINDOW & ACCENT-LEFT \\
\hline a. $\sigma \sigma(\sigma \sigma)$ & $* !$ & $*$ \\
\hline b. $\sigma \sigma(\sigma \sigma)$ & & $* * !$ \\
\hline c. $\sigma \sigma(\sigma \sigma \sigma)$ & & $* * ! *$ \\
\hline d. $\sigma \sigma(\sigma \sigma)$ & & \\
\hline
\end{tabular}

An RSA approach to infixation also has the advantage, then, of providing a general approach to infixation and accent windows, unifying two phenomena that at first glance appear to be unrelated.

3.3 Avoidance of the Midpoint Pathology The Midpoint Pathology (Eisner 1997; Hyde 2012, 2015) is a set of defective predictions arising under the GA approach to alignment. In a Midpoint Pathology effect, an instance of one aligned category, ACat 2 , is consistently drawn to the center of a string or substring of instances of the other aligned category, ACat1. In particular, as (30) illustrates, the string of instances of ACat1 is divided into a number of substrings equal to the number of instances of ACat 2 with an instance of ACat 2 occurring near the center of each substring. Notice that the center orientation is consistent: an aligned object is drawn to the center of a string or substring regardless of its length. ACatl moves to the center of a string of substring regardless of the number of instances of ACat 2 it contains. ${ }^{1}$

\footnotetext{
${ }^{1}$ Incidental occurrence of an aligned object near the center only in strings of certain lengths is not a Midpoint Pathology effect. For example, antepenultimate stress incidentally occurs near the center in forms containing five syllables and forms containing six syllables. Antepenultimate stress, of course, it is not a Midpoint Pathology effect. It is not a
} 
Midpoint Pathology effects

ACat2

a. [ACat1, ACat1, ACat1, ACat1, ACat1, ACat1, ACat1, ACat1, ACat1, ACat1, ACat1, ACat1]

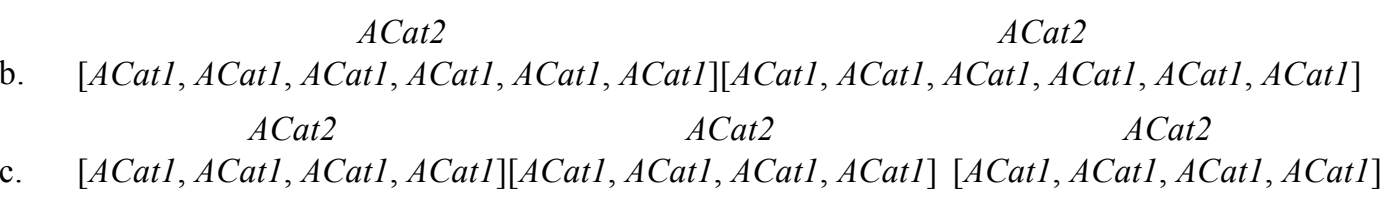

Results like those in (30) are uncontroversially problematic due to the center orientation of ACat1. While orientation with respect to the edges of a string or substring is extremely well attested, orientation with respect to the center of a string or substring is not attested.

Midpoint Pathology effects arise because GA constraints are both distance-sensitive and relationgeneral. They are distance-sensitive in that they assess a greater number of violations for a greater degree of misalignment. They are relation-general in that they assess violations for misalignment regardless of the structural configuration of the misaligned categories. To illustrate, consider the preferences of the GA constraint in (31), which aligns the left edge of every syllable with the left edge of a foot.

Align ( $\sigma, L, F, L, \sigma)$ : The left edge of every syllable coincides with the left edge of some foot. Assess a violation mark for each syllable intervening between misaligned edges.

As (32) demonstrates, when a string of syllables contains a single foot, ALIGN $(\sigma, L)$ draws the foot to the middle of the string, so that the left edge of the foot aligns with the left edge of the medial syllable. Notice that the assessment of violations is distance-sensitive. The left edges of syllables that occur further away from the left edge of the foot incur a greater number of violations than the left edges of syllables that occur closer to the left edge of the foot. Notice also that assessment of violations is relation-general. Misaligned syllables produce violations whether they precede the foot, are contained within the foot, or follow the foot. (Violations produced by syllables preceding the foot are indicated with a "p"; violations produced by syllables contained within the foot are indicated with a "c"; and violations produced by syllables following the foot are indicated with an "f".) The foot is drawn to the center of the string of syllables because the overall misalignment between the foot and every syllable is least in this position.

\begin{tabular}{|r|c|}
\hline & $\operatorname{ALIGN}(\sigma, \mathrm{L})$ \\
\hline a. $[(\sigma \sigma) \sigma \sigma \sigma \sigma \sigma]$ & c ff fff ffff fff!ff ffffff \\
\hline b. $[\sigma(\sigma \sigma) \sigma \sigma \sigma \sigma]$ & p c ff fff ffff ff!fff \\
\hline c. $[\sigma \sigma(\sigma \sigma) \sigma \sigma \sigma]$ & pp p c ff fff ffff! \\
\hline d. $[\sigma \sigma \sigma(\sigma \sigma) \sigma \sigma]$ & ppp pp p c ff fff \\
\hline e. $[\sigma \sigma \sigma \sigma(\sigma \sigma) \sigma]$ & pppp ppp pp p c ff! \\
\hline f. $[\sigma \sigma \sigma \sigma \sigma(\sigma \sigma)]$ & ppppp pppp ppp p!p p c \\
\hline
\end{tabular}

While the example in (32) is something of a toy example, Midpoint Pathology effects are actually so pervasive under GA that GA constraints cannot consistently produce the basic directionality effects for which they have typically been employed in the literature. For example, consider the preferences of the GA constraint ALign (F, L, $\omega, \mathrm{L}, \sigma)$, which aligns the left edges of feet with the left edges of prosodic words.

Align (F, L, $\omega, \mathrm{L}, \sigma)$ : The left edge of every foot coincides with the left edge of some prosodic word. Assess a violation mark for each syllable intervening between misaligned edges.

In the literature, ALIGN (F, L) is typically employed to create a general leftward orientation for feet in forms consisting of a single prosodic word. In an odd-parity prosodic word with a single unfooted syllable, for

pathological effect of any type. This key point has been underappreciated in the recent literature (see, for example, Kager 2012 and, for discussion, Hyde 2015). 
example, Align (F, L) produces a simple pattern of left to right parsing. Producing such simple general directional orientations is one of the essential roles of alignment constraints.

\begin{tabular}{|c|c|}
\hline & $\operatorname{ALIGN}(F, L, \omega, L, \sigma)$ \\
\hline a. $[(\sigma \sigma)(\sigma \sigma)(\sigma \sigma) \sigma]$ & $* * * * * *$ \\
\hline b. $[(\sigma \sigma)(\sigma \sigma) \sigma(\sigma \sigma)]$ & $* * * * * * * !$ \\
\hline c. $[(\sigma \sigma) \sigma(\sigma \sigma)(\sigma \sigma)]$ & $* * * * * * * ! *$ \\
\hline d. $[\sigma(\sigma \sigma)(\sigma \sigma)(\sigma \sigma)]$ & $* * * * * * * ! * *$ \\
\hline
\end{tabular}

In forms with multiple prosodic words, however, as (35) demonstrates, ALIGN (F, L, $\omega, \mathrm{L}, \sigma)$ only produces the desired uniform leftward orientation in the final prosodic word. In nonfinal prosodic words, it results in a type of conflicting directionality. Roughly half of the feet in nonfinal prosodic words do not orient themselves to the left edge of the prosodic word in which they occur; they actually orient themselves to the left edge of the following prosodic word.

\begin{tabular}{|c|c|}
\hline & $\operatorname{ALIGN}(F, L, \omega, L, \sigma)$ \\
\hline a. $\quad[(\sigma \sigma)(\sigma \sigma)(\sigma \sigma) \sigma][(\sigma \sigma)(\sigma \sigma)(\sigma \sigma) \sigma]$ & $\begin{array}{c}{[* * * * *][* * * * * ! *]} \\
(12)\end{array}$ \\
\hline b. $\quad[(\sigma \sigma)(\sigma \sigma) \sigma(\sigma \sigma)][(\sigma \sigma)(\sigma \sigma)(\sigma \sigma) \sigma]$ & $\begin{array}{c}{[* * * *][* * * * * *]} \\
(10)\end{array}$ \\
\hline
\end{tabular}

While the fixed position of the prosodic word boundaries makes this particular case difficult to identify as a Midpoint Pathology effect at first glance, the result emerges due to the combined distance-sensitivity and relation-generality of GA constraints. The overall misalignment between left foot and prosodic word edges is minimized when some of the feet are oriented towards the left edge of the prosodic word that follows rather than the left edge of the prosodic word in which they occur. In particular, the final foot of the first prosodic word in (35a) would have produced four violations if assessment had proceeded with respect to the left edge of the first prosodic word, but it only produces three violations when assessment proceeds with respect to the left edge of the second. The same foot in (35b) would have produced five violations if assessment had proceeded with respect to the left edge of the first prosodic word, but it only produces two violations when assessment proceeds with respect to the left edge of the second. Since assessment can be relative to the second prosodic word, the mixed directionality of candidate (35b) emerges as the winner. This type of evaluation is possible because GA constraints only concern themselves with misalignment; they do not concern themselves with the structural configurations of the misaligned categories.

As demonstrated in (Hyde 2012, 2015, 2016), the RSA formulation avoids the Midpoint Pathology altogether. Because RSA constraints are relation-specific - because they only assess violations for misalignment when the aligned categories appear in the targeted structural configuration-RSA constraints simply cannot produce Midpoint Pathology effects. For example, consider how a leftward foot alignment constraint would have to be formulated under RSA. As (36) indicates, the ALL-FEET-LEFT constraint would prohibit a foot from following a syllable within a prosodic word. It would prohibit misalignment between the left edges of feet and prosodic words, but only when the prosodic word contains the feet.

$$
\begin{aligned}
& \text { ALL-FEET-Left: } *\langle\omega, F, \sigma\rangle /[\ldots \sigma \ldots F \ldots]_{\omega} \\
& \quad \text { Assess a violation mark for every }\langle\omega, F, \sigma\rangle \text { such that } \sigma \text { precedes } F \text { within } \omega .
\end{aligned}
$$

The tableau in (37) shows how ALL-FEET-LEFT produces the desired result for leftward foot alignment even in forms containing multiple prosodic words. Since it only assesses violations for misalignment when the misaligned feet are contained within the prosodic word, a candidate cannot improve its performance when one or more feet in a nonfinal prosodic word is evaluated with respect to the prosodic word that follows. In this case, the final foot of the first prosodic word must be evaluated with respect to the left edge of the first prosodic word, rather than the second. The alignment of the final foot of the first prosodic word, within the first prosodic word, is better with the uniform leftward orientation of (37a). 


\begin{tabular}{|c|c|}
\hline & ALL-FEET-LEFT \\
\hline a. $\quad[(\sigma \sigma)(\sigma \sigma)(\sigma \sigma) \sigma][(\sigma \sigma)(\sigma \sigma)(\sigma \sigma) \sigma]$ & $\begin{array}{c}{[* * * * * *][* * * * * *]} \\
(12)\end{array}$ \\
\hline b. $\quad[(\sigma \sigma)(\sigma \sigma) \sigma(\sigma \sigma)][(\sigma \sigma)(\sigma \sigma)(\sigma \sigma) \sigma]$ & $\begin{array}{c}{[* * * * * * *][* * * * * * !]} \\
(13)\end{array}$ \\
\hline
\end{tabular}

Since the Midpoint Pathology prevents violable GA constraints from producing their essential directionality effects in key contexts, it presents a potentially significant problem for analyses where violable GA constraints play even a minor role. Although the nonviolability of Yu's (2007) GA subcategorization constraints prevents them from producing Midpoint Pathology effects, GA constraints present a serious problem in most contexts where they are violable. While it is possible, it would seem undesirable to have two types of alignment constraints: GA constraints for subcategorization and another type for other uses. Since RSA constraints avoid Midpoint Pathology effects when violable, and since they also produce the desired result when employed as non-violable subcategorization constraints, the most desirable option would seem to be to assume that RSA constraints are the grammar's only alignment constraints.

\section{Conclusion}

An RSA approach to Phonological Subcategorization provides a solid foundation for the analysis of infixation. The RSA approach retains the advantages that earlier Phonological Subcategorization approaches have over alternative approaches. It avoids hyper-infixation; it encompasses cases where infixes attach to prominent positions; and it provides a general analysis of tmesis. The RSA approach has several advantages over GA-based approaches to Phonological Subcategorization. It simplifies the specification of pivot categories while still avoiding hyper-infixation; it provides a uniform, general analysis of infixation and accent windows; and it avoids Midpoint Pathology effects.

\section{References}

Anderson, Stephen. (1992) A-morphous morphology. Cambridge: Cambridge University Press.

Comrie, Bernard. 1976. Irregular stress in Polish and Macedonian. International Review of Slavic Linguistics 1: 227240.

Eisner, Jason. (1997) 'What constraints should OT allow?' Paper presented at the Annual Meeting of the Linguistic Society of America, Chicago. ROA-204.

Green, Thomas Michael (1999) A lexicographic study of Ulwa. Ph.D. dissertation. MIT.

Hyde, Brett (2012) 'Alignment constraints.' Natural Language and Linguistic Theory 30. 789-836.

Hyde, Brett (2015) 'The Midpoint Pathology: What it is and what it isn't.' Ms, Washington University. Rutgers Optimality Archive. http://roa.rutgers.edu/. ROA-1231.

Hyde, Brett (2016) Layering and Directionality: Metrical Stress in Optimality Theory. London: Equinox.

Hyde, Brett, Kenny Hofmeister, and Brooke Husic (2012) Post-Peninitial Accent in Kashaya: An Alternative to Initial Extrametricality. WCCFL 29, 117-124.

Hyde, Brett and Brooke Husic (2012) Ancient Greek Accent Windows. Revista Letras e Letras 28, 29-58.

Inkelas, Sharon (1990) Prosodic constituency in the lexicon. New York: Garland.

Kager, René (2012) Stress in windows: Language typology and factorial typology. Lingua 122. 1454-1493.

Kiparsky, Paul (1986) The phonology of reduplication. Ms., Stanford University.

McCarthy, John and Alan Prince (1986) Prosodic morphology. Ms., UMass and Brandeis.

McCarthy, John and Alan Prince (1993) 'Generalized Alignment' In G. Booij and J. van Marle (eds.), Yearbook of Morphology 1993 (Dordrecht: Kluwer Academics), 79-153

McCarthy, John. (2003) 'OT constraints are categorical', Phonology, 20 (1): 75-138

Prince, Alan and Paul Smolensky (1993) Optimality Theory: Constraint Interaction in generative Grammar (Rutgers University and University of Colorado).

Orgun, Cemil Orhan and Ronald Sprouse (1999) 'From MParse to control: Deriving ungrammaticality', Phonology, 16: 191-224.

Yu, Alan C. L. (2007) A Natural History of Infixation. Oxford: Oxford UP 